九州大学学術情報リポジトリ

Kyushu University Institutional Repository

\title{
Modular Forms and Second Order Ordinary Differential Equations: Applications to Vertex Operator Algebras
}

Kaneko, Masanobu

Faculty of Mathematics, Kyushu University

Nagatomo, Ki yokazu

Department of Pure and Applied Mathematics, Graduate School of Information Science and Technology, Osaka University

Sakai, Yuichi

http://hdl. handle. net/2324/26122

出版情報: Letters in Mathematical Physics. 103 (4), pp.439-453，2013-04. Springer Science+Business Media

バージョン:

権利関係: (C) Springer Science+Business Media Dordrecht 2012 


\title{
Modular Forms and Second Order Ordinary Differential Equations-Applications to Vertex Operator Algebras
}

\author{
Masanobu Kaneko*, Kiyokazu Nagatomo**, and Yuichi Sakai \\ *) Faculty of Mathematics, Kyushu University \\ Fukuoka 819-0395, Japan \\ **) Department of Pure and Applied Mathematics \\ Graduate School of Information Science and Technology \\ Osaka University, Toyonaka, Osaka 560-0043, Japan
}

\begin{abstract}
We study the relation between the Kaneko-Zagier equation and the Mathur-Mukhi-Sen classification, and extend it to the case of solutions with logarithmic terms, which correspond to pseudo-characters of non-rational vertex operator algebras. As an application, we prove a non-existence theorem of rational vertex operator algebras.
\end{abstract}

AMS Subject Classification 2010: Primary 11F11, Secondary 17B69

Key words: Vertex operator algebra, Modular invariance, Modular differential equation

\section{Introduction}

This paper mainly study the Kaneko-Zagier differential equation. We construct exact solutions that correspond to the classification of conformal field theory given by S. D. Mathur, S. Mukhi and A. Sen (MMS classification for short) [6], and extend it to the case of solutions with logarithmic terms.

The MMS classification is based on modular invariance property of the space of characters of simple modules for rational vertex operator algebras. The point of their approach is the use of modular differential equations [5], i.e.

$$
\frac{d^{2} h(\tau)}{d \tau^{2}}+a(\tau) \frac{d h(\tau)}{d \tau}+b(\tau) h(\tau)=0,
$$

where $\tau$ is the variable in the complex upper half-plane, $a(\tau)$ is a constant multiple of the quasimodular Eisenstein series $E_{2}(\tau)$ and $b(\tau)$ is a modular form of weight 4 on the modular group $\Gamma=S L(2, \mathbb{Z})$. Solving (1.1) by the Frobenius method, Mathur, Mukhi and Sen obtained the list of all possible $q$-graded characters and gave the corresponding rational conformal field theories.

The differential equation (1.1) is, as it turns out, equivalent to the differential equation introduced by Kaneko and Zagier [4] for number theoretical motivation; the study of $j$-invariants of supersingular elliptic curves. After the work [4], Kaneko and Koike [3, 2] 
extensively studied this differential equation (the "K-Z equation" for short) and explicitly constructed solutions which are modular or quasimodular forms of various levels and weights.

In this paper, by using these works, we describe exact solutions that correspond to the MMS classification, and develop further their classification and our exact description to the case of solutions with logarithmic terms. In the theory of rational vertex operator algebras, any $q$-graded character of simple module is a holomorphic function (in $q=e^{2 \pi i \tau}$ ). Therefore, these solutions that we find are candidates of pseudo-characters of non-rational vertex operator algebras.

As an application of the existence of solutions with logarithmic terms, we prove a nonexistence theorem of rational vertex operator algebras with two-dimensional space of pseudocharacters.

The paper is organized as follows. In section 2, we recall the concept of pseudo-characters which generalizes $q$-graded characters for a rational vertex operator algebra. We study the $\mathrm{K}-\mathrm{Z}$ equation and the MMS classification as well as its generalization in section 3. The final section is devoted to an application of our main results to the theory of vertex operator algebras.

\section{Pseudo-characters}

\section{$2.1 \quad q$-graded traces of simple modules for vertex operator al- gebras}

Let $V=\bigoplus_{n=0}^{\infty} V_{n}$ be a vertex operator algebra. We write as $n=|v|$ for an element $v$ in $V_{n}$. We say that $V$ satisfies Zhu's finiteness condition if the codimension of the vector subspace that is linearly generated by $a_{(-n)} b(a, b \in V, n \geq 2)$ of $V$ is finite. Suppose that $V$ satisfies Zhu's finiteness condition and let $M$ be a simple $V$-module. Then, we have

$$
M=\bigoplus_{n=0}^{\infty} M_{r+n}, \quad M_{r} \neq 0, \quad M_{r+n}=\left\{m \in M: L_{0} m=(r+n) m\right\}
$$

and $\operatorname{dim}_{\mathbb{C}} M_{r+n}<\infty$ for any nonnegative integer $n$, where $r$ is a complex number called a conformal weight. It is well-known that any conformal weight $r$ of a vertex operator algebra satisfying Zhu's finiteness condition is a rational number ([7]). Let $c_{V}$ be the central charge of $V$. Then $c_{V}$ is also a rational number (see [7]).

We define the $q$-graded trace $\chi_{M}(-, q)$ of a simple module $M$ by

$$
\chi_{M}(v, q)=\operatorname{tr}_{M} o(v) q^{L_{0}-c_{V} / 24}
$$

for any homogeneous $v \in V$, where $o(v)=v_{(|v|-1)}: M_{r+n} \rightarrow M_{r+n}$ for any nonnegative integer $n$. Note that for the vacuum element $v=|0\rangle$, we have

$$
\chi_{M}(q):=\chi_{M}(|0\rangle, q)=\operatorname{tr}_{M} \operatorname{id}_{M} q^{L_{0}-c_{V} / 24}=\sum_{n=0}^{\infty}\left(\operatorname{dim}_{\mathbb{C}} M_{r+n}\right) q^{r+n-c_{V} / 24}
$$

which is called the q-graded character of the simple module $M$. 


\subsection{Modular invariance of the space of $q$-graded characters}

Let $V$ be a rational vertex operator algebra satisfying Zhu's finiteness condition and let $\left\{M^{1}, \ldots, M^{s}\right\}$ be the complete list of inequivalent simple modules. Suppose that an element $v \in V$ is a homogeneous singular element of weight $k=|v|$, i.e. $L_{n} v=0$ for all positive integers $n$ and $v \in V_{k}$, where $\left\{L_{n}, c_{V} \mathrm{id}\right\}_{n \in \mathbb{Z}}$ is the Virasoro algebra associated with $V$.

For any singular vector $v \in V_{k}$, we denote by $\mathbb{M}_{v}$ the vector space linearly spanned by $\chi_{M^{1}}(v, q), \ldots, \chi_{M^{s}}(v, q)$. Then, it is well-known $([8])$ that the vector space $\mathbb{M}_{v}$ is invariant under the action of the slash operator $\left.\right|_{k}$ of the modular group $\Gamma=S L(2, \mathbb{Z})$, which is defined by

$$
\left.f\right|_{k}(\tau)=(c \tau+d)^{-k} f\left(\frac{a \tau+b}{c \tau+d}\right) \quad \text { for }\left(\begin{array}{ll}
a & b \\
c & d
\end{array}\right) \in \Gamma .
$$

Here, $\tau$ is a variable in the upper half-plane and $q=e^{2 \pi i \tau}$.

\subsection{Pseudo-characters of vertex operator algebras}

We recall the definition of one-point functions and the concept of pseudo-characters.

Let $E_{2 k}(k \geq 2)$ be the normalized Eisenstein series of weight $2 k$ on the modular group $\Gamma$ defined by

$$
E_{2 k}(\tau)=\frac{1}{2} \sum_{\substack{m, n \in \mathbb{Z} \\(m, n)=1}} \frac{1}{(m \tau+n)^{2 k}},
$$

so normalized that its Fourier series

$$
E_{2 k}(\tau)=1-\frac{2 k}{B_{2 k}} \sum_{n=1}^{\infty} \sigma_{2 k-1}(n) q^{n}
$$

starts with 1 . Here, $B_{2 k}$ is the Bernoulli number and $\sigma_{2 k-1}(n)$ is the sum of $(2 k-1)$-st power of positive divisors of $n$. Recall that the ring of holomorphic modular forms of integral weight for $\Gamma$ is identified with the polynomial ring $\mathbb{C}\left[E_{4}, E_{6}\right]$.

Definition 2.1. Define the linear space $O_{q}(V)$ as a $\mathbb{C}\left[E_{4}, E_{6}\right]$-submodule of $\mathbb{C}\left[E_{4}, E_{6}\right] \otimes V$ linearly generated by elements

$$
\begin{array}{ll}
u_{(0)} v & (\forall u, v \in V), \\
u_{(-2)} v+\sum_{k=2}^{\infty} 2(2 k-1) \zeta(2 k) E_{2 k}(\tau) \otimes u_{(2 k-2)} v, & (\forall u, v \in V),
\end{array}
$$

where $\zeta(2 k)=\sum_{n=1}^{\infty} 1 / n^{2 k}$ is the value of the Riemann zeta function at $2 k$.

Definition 2.2. Let $\mathcal{H}$ be the upper half-plane. A functional $S(-, \tau): \mathbb{C}\left[E_{4}, E_{6}\right] \otimes V \otimes \mathcal{H} \rightarrow \mathbb{C}$ satisfying the following conditions is called a one-point function on the torus:

i) For any $v \in \mathbb{C}\left[E_{4}, E_{6}\right] \otimes V$, the function $S(v, \tau)$ of $\tau$ is holomorphic in $\mathcal{H}$;

ii) $S\left(\sum_{i} f_{i}(\tau) \otimes v_{i}, \tau\right)=\sum_{i} f_{i}(\tau) S\left(v_{i}, \tau\right)$ for any $v_{i} \in V$ and $f_{i}(\tau) \in \mathbb{C}\left[E_{4}, E_{6}\right]$; 
iii) $S(v, \tau)=0$ for all $v \in O_{q}(V)$;

iv) For any $v \in V_{n}$, one has

$$
S\left(L_{-2} v, \tau\right)=\partial S(v, \tau)+\sum_{k=2}^{\infty} 2 \zeta(2 k) E_{2 k}(\tau) S\left(L_{2 k-2} u, \tau\right),
$$

where we define $\partial$ by

$$
\partial S(v, \tau)=\partial_{n} S(v, \tau)=2 \pi i \frac{d}{d \tau} S(v, \tau)+\frac{n \pi^{2}}{3} E_{2}(\tau) S(v, \tau)
$$

for any homogeneous $v \in V_{n}$ and extend it by linearity. Here, the function $E_{2}(\tau)$ is the (quasimodular) Eisenstein series of weight 2 defined by

$$
E_{2}(\tau):=1-24 \sum_{n=1}^{\infty} \sigma_{1}(n) q^{n} .
$$

We denote the space of one-point functions (on the torus) by $\mathcal{e}(V)$.

Definition 2.3. Let $V$ be a vertex operator algebra. For any one-point function $S(-, \tau): V \rightarrow$ $\mathbb{C}$, the function $S(|0\rangle, \tau)$ is called a pseudo-character of $V$.

The following theorem is well-known.

Theorem 2.4. Let $V$ be a rational vertex operator algebra satisfying Zhu's finiteness condition. Then the space of pseudo-characters of $V$ has a basis $\left\{\chi_{M^{1}}(-), \ldots, \chi_{M^{r}}(-)\right\}$, where $\left\{M_{1}, \ldots, M_{r}\right\}$ is the complete list of inequivalent simple modules. In other words, any pseudo-character is a linear combination of trace functions of simple modules.

\subsection{Differential equations for pseudo-characters}

The space $\mathbb{M}_{v}\left(v \in V_{n}\right)$ is invariant under the action of the slash operator $\left.\right|_{n}$. There is a canonical way to obtain ordinary differential equation of which the space of solutions is $\mathbb{M}_{v}$. To see this, we prepare several preliminary definitions and lemmas on derivatives of modular forms.

Definition 2.5. Introduce the first order differential operator $\mathcal{D}$ acting on modular forms by

$$
\mathcal{D}=q \frac{d}{d q}-\frac{1}{12} E_{2}(\tau) \cdot w,
$$

where $w(f)=k f$ if $f$ is of weight $k$.

The following lemma is well-known.

Lemma 2.6. Let $f$ be a modular form of weight $k$ on $\Gamma$ or its subgroup. Then $\mathcal{D} f$ is modular of weight $k+2$ on the same group. 
Definition 2.7. Let $\left\{f_{1}, \ldots, f_{n}\right\}$ be a set of linearly independent modular forms of weight $k$, and let

$$
W_{i}=\operatorname{det}\left(\begin{array}{ccc}
f_{1} & \ldots & f_{n} \\
\mathcal{D} f_{1} & \ldots & \mathcal{D} f_{n} \\
\vdots & \ldots & \vdots \\
\mathcal{D}^{i-1} f_{1} & \ldots & \mathcal{D}^{i-1} f_{n} \\
\mathcal{D}^{i+1} f_{1} & \ldots & \mathcal{D}^{i+1} f_{n} \\
\vdots & \ldots & \vdots \\
\mathcal{D}^{n} f_{1} & \ldots & \mathcal{D}^{n} f_{n}
\end{array}\right)
$$

for any integer $0 \leq i \leq n$, and introduce functions $\phi_{i}(0 \leq i \leq n-1)$ by

$$
\phi_{i}(\tau)=(-1)^{n-i} W_{i} / W_{n} .
$$

Lemma 2.8. The functions $\phi_{i}(\tau)(0 \leq i \leq n-1)$ are modular forms of weight $2(n-i)$.

Proof. By the definition of determinant and Lemma 2.6, it follows that $W_{i}(0 \leq i \leq n-1)$ is a modular form of weight

$$
k+(k+2)+\cdots+(k+2 n)-(k+2 i)=n k+n(n+1)-2 i
$$

and thus $\phi_{i}(\tau)(0 \leq i \leq n-1)$ is a modular form of weight

$$
n k+n(n+1)-2 i-n k-n(n+1)+2 n=2(n-i) .
$$

\subsection{Two-dimensional case}

We study the case that the vector space $\mathbb{M}_{v}$ is two-dimensional. Let $\chi_{1}(\tau)=\chi_{1}(v, \tau)$ and $\chi_{1}(\tau)=\chi_{2}(v, \tau)$ form a basis of the vector space $\mathbb{M}_{v}$. Then, $\chi_{1}(\tau)$ and $\chi_{2}(\tau)$ are solutions of the second order ordinary differential equation

$$
f^{\prime \prime}(\tau)-\frac{\left(\chi_{1}(\tau) \chi_{2}^{\prime \prime}(\tau)-\chi_{2}(\tau) \chi_{1}^{\prime \prime}(\tau)\right)}{W(\tau)} f^{\prime}(\tau)+\frac{\left(\chi_{1}^{\prime}(\tau) \chi_{2}^{\prime \prime}(\tau)-\chi_{2}^{\prime}(\tau) \chi_{1}^{\prime \prime}(\tau)\right)}{W(\tau)} f(\tau)=0,
$$

where $W(\tau)$ is a wronskian, i.e. $W(\tau)=\chi_{1}(\tau) \chi_{2}^{\prime}(\tau)-\chi_{2}(\tau) \chi_{1}^{\prime}(\tau)$, and ' means the derivative D. Let

$$
a_{1}(\tau)=-\frac{\chi_{1}(\tau) \chi_{2}^{\prime \prime}(\tau)-\chi_{2}(\tau) \chi_{1}^{\prime \prime}(\tau)}{W(\tau)}, \quad a_{2}(\tau)=\frac{\chi_{1}^{\prime}(\tau) \chi_{2}^{\prime \prime}(\tau)-\chi_{2}^{\prime}(\tau) \chi_{1}^{\prime \prime}(\tau)}{W(\tau)} .
$$

Then, the modular invariance property of $\chi_{1}(\tau)$ and $\chi_{2}(\tau)$ implies that $a_{1}(\tau)$ and $a_{2}(\tau)$ are (meromorphic) modular forms of weights 2 and 4, respectively.

Proposition 2.9. Let $V$ be a vertex operator algebra satisfying Zhu's finiteness condition, and suppose that the space of pseudo-characters of $V$ is two-dimensional. Let $\left\{\chi_{1}(\tau), \chi_{2}(\tau)\right\}$ be a basis of the space of pseudo-characters of $V$, then $a_{1}(\tau)$ and $a_{2}(\tau)$ given by (2.16) are (meromorphic) modular forms of weight 2 and 4 , respectively. 


\section{$3 \quad$ MMS theory and Kaneko-Zagier equation}

\subsection{The Kaneko-Zagier equation}

Let $k$ be a rational number. The so-called Kaneko-Zagier equation (K-Z equation for short) is the second order ordinary differential equation

$$
f^{\prime \prime}(\tau)-\frac{k+1}{6} E_{2}(\tau) f^{\prime}(\tau)+\frac{k(k+1)}{12} E_{2}^{\prime}(\tau) f(\tau)=0,
$$

where

$$
'=\frac{1}{2 \pi i} \frac{d}{d \tau}=q \frac{d}{d q}
$$

A particular solution of this equation, which is a modular form of weight $k$, was studied in [4] from a viewpoint of number theory (in relation to $j$-invariants of supersingular elliptic curves), and then various modular or quasimodular form solutions were found and described in [3] and [2].

Suppose $f(\tau)$ is a solution of $(\sharp)_{k}$ that is modular of weight $k$ (on some congruence subgroup). Then, the quotient $f(\tau) / \eta(\tau)^{2 k}$ is a modular function (of weight 0 ), where

$$
\eta(\tau)=q^{1 / 24} \prod_{n=1}^{\infty}\left(1-q^{n}\right)
$$

is the Dedekind eta function. What Mathur et al. did in [6] is, in our framework, to list all possible solutions $f(\tau)$ with which the quotient $f(\tau) / \eta(\tau)^{2 k}$ has positive integral Fourier coefficients. Let us reformulate their argument in our setting.

Put $g(\tau)=f(\tau) / \eta(\tau)^{2 k}$. By the well-known formulas

$$
\eta^{\prime}(\tau)=\frac{1}{24} \eta(\tau) E_{2}(\tau) \quad \text { and } \quad E_{2}^{\prime}(\tau)=\frac{1}{12}\left(E_{2}(\tau)^{2}-E_{4}(\tau)\right)
$$

where

$$
E_{4}(\tau)=1+240 \sum_{n=1}^{\infty}\left(\sum_{d \mid n} d^{3}\right) q^{n}=1+240 q++2160 q^{2}+6720 q^{3}+\cdots
$$

is the Eisenstein series of weight 4 on $\Gamma$, the equation $(\sharp)_{k}$ is transformed to the equation for $g(\tau)$ as

$$
g^{\prime \prime}(\tau)-\frac{1}{6} E_{2}(\tau) g^{\prime}(\tau)-\frac{k(k+2)}{144} E_{4}(\tau) g(\tau)=0 .
$$

Now, we find a solution $g(\tau)$ of the form

$$
g(\tau)=q^{\alpha}\left(1+\sum_{n=1}^{\infty} a_{n} q^{n}\right)
$$

where $\alpha$ is a rational number and $a_{n}(n \in \mathbb{N})$ are positive integers. 
Remark 3.1. Recall that any $q$-graded character of a vertex operator algebra is of the form (3.3) (with $\alpha=r-c_{V} / 24$ ), because if $V$ satisfies Zhu's finiteness condition, the conformal weight $r$ and the central charge $c_{V}$ are rational numbers. Moreover, because the coefficients of a $q$-graded character is dimensions of the homogeneous spaces, numbers $a_{n}$ are non-negative integers. The lowest part $M_{0}$ of $V$-modules is assumed to be one-dimensional.

Before proceeding further, we state a duality between the solution spaces of $(\sharp)_{k}$ and $(\sharp)_{-2-k}$, from which we can restrict our discussion to the case $k>-1$.

Proposition 3.2. Suppose that $f$ is a solution of $(\sharp)_{k}$. Then, $f / \eta^{4 k+4}$ is a solution of $(\sharp)_{-2-k}$.

Proof. The proof is straightforward computation with (3.2).

The vector spaces $\mathcal{V}_{k}$ and $\mathcal{V}_{-2-k}$ of solutions of $(\sharp)_{k}$ and $(\sharp)_{-2-k}$ respectively are mutually isomorphic by the map

$$
\mathcal{V}_{k} \ni f \mapsto f / \eta^{4 k+4} \in \mathcal{V}_{-2-k}
$$

and its inverse

$$
\mathcal{V}_{-2-k} \ni g \mapsto g / \eta^{4(-2-k)+4}=g \eta^{4 k+4} \in \mathcal{V}_{k} .
$$

From this duality and the following equality

$$
f / \eta^{2 k}=\left(f / \eta^{4+4 k}\right) / \eta^{2(-2-k)},
$$

we conclude that, in order to study weight 0 functions $f / \eta^{2 k}$ for $f \in \mathcal{V}_{k}$, it is enough to consider the case $k \geq-2-k$, i.e. $k \geq-1$. The equation $(\sharp)_{k}$ with $k=-1$ becomes the trivial one $f^{\prime \prime}=0$. Therefore, we assume $k>-1$ in the following.

\subsection{Mathur-Mukhi-Sen classification}

We resume our search for solutions $g(\tau)$ of the form (3.3) with a rational $\alpha$ and positive integral $a_{n}$. Substituting (3.3) into $(b)_{k}$ and looking at the coefficient of the lowest degree, we find that $\alpha$ satisfies the quadratic equation

$$
\alpha^{2}-\frac{1}{6} \alpha-\frac{k(k+2)}{144}=0,
$$

i.e. $\alpha=-k / 12,(k+2) / 12$.

We first consider the case $\alpha=-k / 12$. Rewrite the Fourier series of Eisenstein series as

$$
E_{k}(\tau)=1+\sum_{n=1}^{\infty} e_{k, n} q^{n} \quad(k=2,4)
$$

and set $e_{k, 0}=1$. Then, looking at the coefficients of $q^{\alpha+n}$ of $(b)_{k}$, we find the relation

$$
(n+\alpha)^{2} a_{n}-\frac{1}{6} \sum_{i=0}^{n} e_{2, i}(n-i+\alpha) a_{n-i}-\frac{k(k+2)}{144} \sum_{i=0}^{n} e_{4, i} a_{n-i}=0,
$$


or equivalently $(\alpha=-k / 12)$

$$
n\left(n-\frac{k+1}{6}\right) a_{n}=\frac{1}{6} \sum_{i=1}^{n}\left\{e_{2, i}\left(n-i-\frac{k}{12}\right)+\frac{k(k+2)}{24} e_{4, i}\right\} a_{n-i}
$$

for all positive integers $n$. In particular, for $n=1$ we have

$$
\left(1-\frac{k+1}{6}\right) a_{1}=\frac{1}{6}(2 k+10 k(k+2)),
$$

i.e.

$$
a_{1}=\frac{2 k(5 k+11)}{5-k} .
$$

Note that when $k=5$, we know from [3] that the equation $(\sharp)_{k}$ has no modular solutions (it has a quasimodular solution that is studied in $\S 3.3$ ). Hence, we can suppose $k \neq 5$.

Now, by our assumption, the quantity

$$
m:=\frac{2 k(5 k+11)}{5-k}\left(=a_{1}\right)
$$

is a positive integer, and hence the equation

$$
10 k^{2}+(22+m) k-5 m=0
$$

in $k$ (which is rational) has an integral square discriminant:

$$
(m+2)^{2}+200 m=\ell^{2} .
$$

This equation can be rewritten as

$$
(m+\ell+122)(m-\ell+122)=14400 .
$$

This shows that integers $m \pm \ell+122$ are divisors of 14400 , and it is not difficult to list all possible solutions $(m, \ell)$. For such $(m, \ell)$, we find the following values of $k$ with which the coefficient $a_{1}$ is a positive integers:

$$
k=\frac{1}{5}, \frac{1}{2}, 1, \frac{7}{5}, 2, \frac{5}{2}, \frac{13}{5}, 3, \frac{16}{5}, \frac{17}{5}, \frac{7}{2}, \frac{19}{5}, 4, \frac{41}{10}, \frac{21}{5}, \frac{22}{5}, \frac{9}{2}, \frac{23}{5}, \frac{47}{10}, \frac{24}{5}, \frac{49}{10} .
$$

Setting $n=2$ in (3.7), we have

$$
(11-k) a_{2}=\left(5 k^{2}+11 k-12\right) a_{1}+45 k^{2}+93 k .
$$

Then among the values of $k$ in (3.11), the followings give positive integral $a_{2}$ :

$$
k=\frac{1}{5}, \frac{1}{2}, 1, \frac{7}{5}, 2, \frac{13}{5}, 3, \frac{7}{2}, \frac{19}{5}, 4, \frac{22}{5}, \frac{47}{10} .
$$

We find by using (3.7) that $k=22 / 5$ gives non-integral $a_{4}$ and $k=47 / 10$ gives non-integral $a_{3}$, so the final candidates for $k$ are

$$
k=\frac{1}{5}, \frac{1}{2}, 1, \frac{7}{5}, 2, \frac{13}{5}, 3, \frac{7}{2}, \frac{19}{5}, 4 .
$$


Remark 3.3. The list of $k$ in (3.12) coincides with Table 1 in [6] where $(c=) c_{V}=2 k$.

For each $k$ in (3.12), the explicit solution $g(\tau)=f(\tau) / \eta(\tau)^{2 k}$ of $(b)_{k}$ can be obtained by using results in [2] and [3]. To describe this, we need the following functions. Note that we use a slightly different notation from that in [2].

Let

$$
E_{2}^{(2)}(\tau):=2 E_{2}(2 \tau)-E_{2}(\tau)=1+24 \sum_{n=1}^{\infty}\left(\sum_{\substack{d \mid n \\ d: \text { odd }}} d\right) q^{n}=1+24 q+24 q^{2}+96 q^{3}+\cdots
$$

be the Eisenstein series of weight 2 and level 2, and

$$
E_{1}^{(3)}(\tau)=1+6 \sum_{n=1}^{\infty}\left(\sum_{d \mid n}\left(\frac{d}{3}\right)\right) q^{n}=1+6 q+6 q^{3}+6 q^{4}+\cdots
$$

the Eisenstein series of weight 1 and level 3, where $\left(\frac{d}{3}\right)$ is the Legendre character. Further, let

$$
\begin{aligned}
& \Delta_{3}^{(3)}(\tau)=\frac{\eta(3 \tau)^{9}}{\eta(\tau)^{3}}=q+3 q^{2}+9 q^{3}+13 q^{4}+\cdots \\
& \Delta_{2}^{(4)}(\tau)=\frac{\eta(4 \tau)^{8}}{\eta(2 \tau)^{4}}=\sum_{\substack{n \geq 1 \\
n: \text { odd }}}\left(\sum_{d \mid n} d\right) q^{n}=q+4 q^{3}+6 q^{5}+8 q^{7}+\cdots
\end{aligned}
$$

be the cusp form of weight 3 and level 3, and of weight 2 and level 4 , respectively. We also use the theta series

$$
\theta_{3}(\tau):=\sum_{n \in \mathbb{Z}} q^{n^{2}}=1+2 q+2 q^{4}+2 q^{9}+2 q^{16}+\cdots
$$

and

$$
\theta_{2}(\tau):=\frac{1}{2} \sum_{n \in \mathbb{Z}} q^{\left(n+\frac{1}{2}\right)^{2}}=q^{\frac{1}{4}}\left(1+q^{2}+q^{6}+q^{12}+q^{20}+\cdots\right) .
$$

Finally, we introduce the Ramanujan-Rogers modular functions of level 5:

$$
\begin{aligned}
& \phi_{1}(\tau)=q^{-1 / 60} \prod_{n=0}^{\infty} \frac{1}{\left(1-q^{5 n+1}\right)\left(1-q^{5 n+4}\right)}, \\
& \phi_{2}(\tau)=q^{11 / 60} \prod_{n=0}^{\infty} \frac{1}{\left(1-q^{5 n+2}\right)\left(1-q^{5 n+3}\right)} .
\end{aligned}
$$

For each $k$ in (3.12), we denote by $g_{k}(\tau)$ the (unique) solution of $(b)_{k}$ of the form (3.3) with $\alpha=-k / 12$. 
Theorem 3.4. For the values of $k$ in (3.12), the functions $g_{k}(\tau)$ are given by

$$
\begin{aligned}
& g_{1 / 5}(\tau)=\phi_{1}(\tau), \quad g_{1 / 2}(\tau)=\frac{\theta_{3}(\tau)}{\eta(\tau)}, \quad g_{1}(\tau)=\frac{E_{1}^{(3)}(\tau)}{\eta(\tau)^{2}}, \quad g_{7 / 5}(\tau)=\phi_{1}(\tau)^{7}+7 \phi_{1}(\tau)^{2} \phi_{2}(\tau)^{5}, \\
& g_{2}(\tau)=\frac{E_{2}^{(2)}(\tau)}{\eta(\tau)^{4}}, \quad g_{13 / 5}(\tau)=\phi_{1}(\tau)^{13}+39 \phi_{1}(\tau)^{8} \phi_{2}(\tau)^{5}-26 \phi_{1}(\tau)^{3} \phi_{2}(\tau)^{10}, \\
& g_{3}(\tau)=\frac{E_{1}^{(3)}(\tau)^{3}+54 \Delta_{3}^{(3)}(\tau)}{\eta(\tau)^{6}}, \quad g_{7 / 2}(\tau)=\frac{\theta(\tau)^{7}+112 \Delta_{2}^{(4)}(\tau) \theta(\tau)^{3}}{\eta(\tau)^{7}}, \\
& g_{19 / 5}(\tau)=\phi_{1}(\tau)^{19}+171 \phi_{1}(\tau)^{14} \phi_{2}(\tau)^{5}+247 \phi_{1}(\tau)^{9} \phi_{2}(\tau)^{10}-57 \phi_{1}(\tau)^{4} \phi_{2}(\tau)^{15}, \\
& g_{4}(\tau)=\frac{E_{4}(\tau)}{\eta(\tau)^{8}} .
\end{aligned}
$$

Remark 3.5. Clearly, the Fourier coefficients of $g_{k}(\tau)$ in the theorem are integers, and the positivity of the coefficients are immediate from the definitions for $g_{1 / 5}, g_{1 / 2}, g_{7 / 5}, g_{2}, g_{7 / 2}$ and $g_{4}$. For $g_{1}$ and $g_{3}$, the positivity follows from the positivity of the coefficients of $E_{1}^{(3)}(\tau)$ and $\Delta_{3}^{(3)}(\tau)$ which was shown in the proof of Theorem 3 in [3]. The rest, for $g_{13 / 5}$ and $g_{19 / 5}$, at the moment, we are not able to show the positivity directly. It is enough to show that the function $\phi_{1}^{5}-\phi_{2}^{5}$ has positive coefficients, which is plausible from numerical experiments.

\subsection{Beyond MMS classification}

Next, we consider the case $\alpha=(k+2) / 12$. In this case, instead of $(3.7)$, we have

$$
n\left(n+\frac{k+1}{6}\right) a_{n}=\frac{1}{6} \sum_{i=1}^{n}\left\{e_{2, i}\left(n-i+\frac{k+2}{12}\right)+\frac{k(k+2)}{24} e_{4, i}\right\} a_{n-i}
$$

and in particular for $n=1$

$$
\left(1+\frac{k+1}{6}\right) a_{1}=\frac{1}{6}(-2(k+2)+10 k(k+2)),
$$

i.e.

$$
a_{1}=\frac{2(k+2)(5 k-1)}{k+7}
$$

We require that this quantity is a positive integer, and obtain as before, a finite list (22 in total) of possible values of $k$. Among them, the values that give positive integral $a_{2}$ that is determined by $(n=2$ of $(3.13))$

$$
(k+13) a_{2}=\left(5 k^{2}+9 k-14\right) a_{1}+45 k^{2}+87 k-6
$$

are

$$
k=\frac{1}{5}, \frac{1}{2}, 1,2,3,5,8,11,17,23,29,53,113 .
$$

For $k=\frac{1}{5}, \frac{1}{2}, 1,2,3,8$, we know from [3] and [2] that there are corresponding modular functions as solutions of $(b)_{k}$. They are described as follows. Denote by $h_{k}(\tau)$ the (unique) solution of $(b)_{k}$ of the form (3.3) with $\alpha=(k+2) / 12$. 
Theorem 3.6. For $k=\frac{1}{5}, \frac{1}{2}, 1,2,3,8$, the functions $h_{k}(\tau)$ are given by

$$
\begin{gathered}
h_{1 / 5}(\tau)=\phi_{2}(\tau), \quad h_{1 / 2}(\tau)=\frac{\theta_{2}(\tau)}{\eta(\tau)}, \quad h_{1}(\tau)=\frac{\eta(3 \tau)^{3}}{\eta(\tau)^{3}}, \quad h_{2}(\tau)=\frac{\eta(2 \tau)^{8}}{\eta(\tau)^{8}}, \\
h_{3}(\tau)=\frac{E_{1}^{(3)}(\tau) \eta(3 \tau)^{6}}{\eta(\tau)^{8}}, \quad h_{8}(\tau)=\frac{E_{2}^{(2)}(\tau) \eta(2 \tau)^{24}}{\eta(\tau)^{28}}
\end{gathered}
$$

which are respectively of level $5,4,3,2,3$, and 2 .

Remark 3.7. It is proved that the Fourier coefficients of these functions are positive integers.

For the remaining values of $k$ in the list (3.16), the solutions $h_{k}(\tau)$ are not modular but "quasimodular". Let $f_{k}(\tau)$ be the normalized quasimodular solution of $(\sharp)_{k}$ given in Theorem 2 in [3]. Then, for $k=5,11,17,23,29,53,113$, we have $h_{k}(\tau)=f_{k}(\tau) / \eta(\tau)^{2 k}$. Apart from the function

$$
h_{5}(\tau)=\frac{1}{240} \frac{E_{4}^{\prime}(\tau)}{\eta(\tau)^{10}}
$$

the explicit formula in [3] gives no clue how to prove the positivity of coefficients of $h_{k}(\tau)$. For instance, we are unable to prove the positivity of the coefficients of

$$
h_{11}(\tau)=\frac{1}{462}\left(\eta(\tau)^{2}-\frac{1}{240} \frac{E_{4}^{\prime}(\tau) E_{6}(\tau)}{\eta(\tau)^{22}}\right)=q^{13 / 12}+78 q^{25 / 12}+2509 q^{37 / 12}+\cdots .
$$

There is one more aspect concerning to these quasimodular solutions. Let us consider solutions of $(b)_{k}$ of the form

$$
\begin{aligned}
g & =g_{1} \log q+g_{2}, \\
g_{1} & =q^{\alpha}\left(1+\sum_{n=1}^{\infty} a_{n} q^{n}\right), \quad g_{2}=q^{\beta} \sum_{n=0}^{\infty} b_{n} q^{n} .
\end{aligned}
$$

Substituting (3.17) into $(b)_{k}$, we obtain

$$
\left(g_{1}^{\prime \prime}-\frac{1}{6} E_{2} g_{1}^{\prime}-\frac{k(k+2)}{144} E_{4} g_{1}\right) \log q+2 g_{1}+g_{2}^{\prime \prime}-\frac{1}{6} E_{2}\left(g_{1}+g_{2}^{\prime}\right)-\frac{k(k+2)}{144} E_{4} g_{2}=0,
$$

and we see that $g_{1}$ is a solution of $(b)_{k}$. Hence, if we require the coefficients $a_{n}$ being positive integers, the weight parameter $k$ must be in one of the lists (3.12) and (3.16).

However, we know from [3] and [2] that the only values of $k$ in the lists that allow solutions with $\log q$ terms are $k=5,11,17,23,29,53,113$. More explicitly, it is shown in the final section of [3] that if we write the quasimodular solution $f_{k}(\tau)$ of $(\sharp)_{k}$ (which is proved to be non-zero) as

$$
f_{k}(\tau)=f_{k}^{(1)}(\tau) E_{2}(\tau)+f_{k}^{(2)}(\tau)
$$

where $f_{k}^{(1)}(\tau)$ and $f_{k}^{(2)}(\tau)$ are modular forms of weights $k-1$ and $k+1$ respectively, then the function

$$
f_{k}(\tau) \log q+12 f_{k}^{(1)}(\tau)
$$

is a solution of $(\sharp)_{k}$.

Remark 3.8. When $k$ is a natural number satisfying $k \equiv 5 \bmod 6$, the differential equation $(\sharp)_{k}$ always has a quasimodular solution, and hence a solution of the form (3.19) with $f_{k}(\tau) \neq 0$. 


\subsection{The MMS equation and the $\mathrm{K}-\mathrm{Z}$ equation}

In this section, we reveal a relationship of the MMS equation to the K-Z equation.

Recall that the MMS equation is

$$
\frac{d^{2} g}{d \tau^{2}}(\tau)-\frac{1}{3} \pi i E_{2}(\tau) \frac{d g}{d \tau}(\tau)+\mu \pi^{2} E_{4}(\tau) g(\tau)=0 .
$$

The solution in question is of the form

$$
g(\tau)=q^{\alpha}\left(\sum_{n=0}^{\infty} g_{n} q^{n}\right) .
$$

Substituting (3.21) into (3.20), we find

$$
\mu=4 \alpha^{2}-\frac{2}{3} \alpha
$$

Let $V=\bigoplus_{n=0}^{\infty} V_{n}$ be a vertex operator algebra satisfying Zhu's finiteness condition and $\ell=\operatorname{dim} V / C_{2}(V)-1$, where $C_{2}(V)$ is the vector space linearly spanned by elements $a_{(-2)} b(a, b \in V)$. Note that the quotient space $V / C_{2}(V)$ is finite-dimensional because of Zhu's finiteness condition. Then, it was proved by M. Gaberdiel and A. Neitzke [1] that

$$
\operatorname{ch}_{V} q^{L_{0}}=\sum_{n=0}^{\infty}\left(\operatorname{dim} V_{n}\right) q^{n} \leq \prod_{n=1}^{\infty}\left(1+q^{n}\right)^{\ell} \quad\left(\ell=\operatorname{dim} V / C_{2}(V)-1\right) .
$$

Now, the right-hand side of $(3.23)$ converges for $0<|q|<1$ and clearly

$$
\operatorname{ch}_{V} q^{L_{0}} \sim 1
$$

as $\tau \rightarrow i \infty$. Therefore, we find

$$
\operatorname{ch}_{V} q^{L_{0}-c / 24} \sim q^{-c / 24}
$$

and thus we have $\alpha=-c / 24$. By solving (3.22), we find

$$
\alpha=\frac{1 \pm \sqrt{1+36 \mu}}{12} .
$$

Because $\alpha=-c / 24$ is a rational number, the number $1+36 \mu$ must be a perfect square, say, $1+36 \mu=(k+1)^{2}$. Then, the MMS equation (3.20) becomes

$$
g^{\prime \prime}(\tau)-\frac{1}{6} E_{2}(\tau) g^{\prime}(\tau)+\frac{k(k+2)}{144} E_{4}(\tau) g(\tau)=0 .
$$

\section{Application to non-rational vertex operator alge- bras}

We give an application of the discussions in $\S 3.3$. 
Theorem 4.1. Let $V$ be a vertex operator algebra satisfying Zhu's finiteness condition. Suppose that the space of pseudo-characters is two-dimensional. If the value of the central charge $c$ is given by $c=2 k$, or $-4-2 k$ with $k \equiv 5(\bmod 6), k \in \mathbb{N}$, the vertex operator algebra $V$ is not rational.

Proof. Suppose on the contrary that $V$ is rational. Then, it is known that any pseudocharacter is a linear combination of $q$-graded characters of simple modules, and that these $q$-graded characters are holomorphic functions of $q$ on the domain $|q|<1$ ([8]). However, for $k$ satisfying the condition in the theorem, the equations $(\sharp)_{k}$ have solutions involving $\log q$ as shown in $\S 3.3$.

Conjecture. For the values of $c=2 k,-4-2 k$ with $k=5,11,17,23,29,53,113$, there is a non-rational vertex operator algebra $V$ with central charge $c$.

\section{References}

1. Gaberdiel, M. and Neitzke, A.: Rationality, quasirationality and finite W-algebras, Comm. Math. Phys. 238, no. 1-2, 305-331(2003).

2. Kaneko, M.: On Modular forms of weight $(6 n+1) / 5$ satisfying a certain differential equation, Number Theory, Developments in Mathematics, 15, 97-102 (2006).

3. Kaneko, M. and Koike, M.: On modular forms arising from a differential equations of hypergeometric type, Ramanujan Journal, 7,145-164 (2003).

4. Kaneko, M. and Zagier, D.: Supersingular $j$-invariants, hypergeometric series, and Atkin's orthogonal polynomials, AMS/IP Studies in Advanced Mathematics, 7, 97-126 (1998).

5. Mason, G.: Vector-valued modular forms and linear differential operators, Int. J. Number Theory, 3, no. 3, 377-390 (2007).

6. Mathur, S. D., Mukhi, S., and Sen, A.: On the classification of rational conformal field theories, Phys. Letter B, 213, No.3, 303-308 (1988).

7. Miyamoto, M.: Modular invariance of vertex operator algebras satisfying $C_{2^{-}}$ cofiniteness, Duke Mathematical Journal, 122, 51-91 (2004).

8. Zhu, Y.: Modular invariance of characters of vertex operator algebras, Journal of the American Mathematical Society, 9, No. 1, 237-302 (1996). 